University of Nebraska - Lincoln

DigitalCommons@University of Nebraska - Lincoln

Public Health Resources

Public Health Resources

2005

Rat Sinusoidal Liver Endothelial cells (SECs) Produce Pro-Fibrotic Factors in Response to Adducts Formed from the Metabolites of Ethanol

\author{
Geoffrey M. Thiele \\ University of Nebraska Medical Center, Department of Internal Medicine, 983025 Nebraska Medical \\ Center, Omaha, NE 68198-3025, USA \\ Michael J. Duryee \\ University of Nebraska Medical Center, Department of Internal Medicine, 983025 Nebraska Medical \\ Center, Omaha, NE 68198-3025, USA \\ Thomas L. Freeman \\ University of Nebraska Medical Center, Department of Internal Medicine, 983025 Nebraska Medical \\ Center, Omaha, NE 68198-3025, USA \\ Michael F. Sorrell \\ University of Nebraska Medical Center, Department of Internal Medicine, 983025 Nebraska Medical \\ Center, Omaha, NE 68198-3025, USA \\ Monte S. Willis \\ University of North Carolina-Chapel Hill, Department of Pathology and Laboratory Medicine CB \#7525, \\ Glaxo Research Lahoratories Rm 148, Chapel Hill, NC 27599-7525, USA

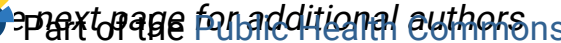

Thiele, Geoffrey M.; Duryee, Michael J.; Freeman, Thomas L.; Sorrell, Michael F.; Willis, Monte S.; Tuma, Dean J.; and Klassen, Lynell W., "Rat Sinusoidal Liver Endothelial cells (SECs) Produce Pro-Fibrotic Factors in Response to Adducts Formed from the Metabolites of Ethanol" (2005). Public Health Resources. 78. https://digitalcommons.unl.edu/publichealthresources/78

This Article is brought to you for free and open access by the Public Health Resources at DigitalCommons@University of Nebraska - Lincoln. It has been accepted for inclusion in Public Health Resources by an authorized administrator of DigitalCommons@University of Nebraska - Lincoln. 
Authors

Geoffrey M. Thiele, Michael J. Duryee, Thomas L. Freeman, Michael F. Sorrell, Monte S. Willis, Dean J. Tuma, and Lynell W. Klassen 


\title{
Rat sinusoidal liver endothelial cells (SECs) produce pro-fibrotic factors in response to adducts formed from the metabolites of ethanol ${ }^{\text {th }}$
}

\author{
Geoffrey M. Thiele ${ }^{\mathrm{a}, \mathrm{b}, \mathrm{c}, *}$, Michael J. Duryee ${ }^{\mathrm{a}, \mathrm{b}}$, Thomas L. Freeman ${ }^{\mathrm{a}, \mathrm{b}}$, \\ Michael F. Sorrell ${ }^{\mathrm{a}}$, Monte S. Willis ${ }^{\mathrm{d}}$, Dean J. Tuma ${ }^{\mathrm{a}, \mathrm{b}}$, Lynell W. Klassen ${ }^{\mathrm{a}, \mathrm{b}}$ \\ ${ }^{a}$ University of Nebraska Medical Center, Department of Internal Medicine, \\ 983025 Nebraska Medical Center, Omaha, NE 68198-3025, USA \\ ${ }^{\mathrm{b}}$ Veterans Administration Alcohol Research Center, Omaha Veterans Administration Medical Center, \\ 4101 Woolworth Avenue, Omaha, NE 68105, USA \\ ${ }^{\mathrm{c}}$ University of Nebraska Medical Center, Department of Pathology and Microbiology, \\ 986495 Nebraska Medical Center, Omaha, NE 68198-6495, USA \\ ${ }^{\mathrm{d}}$ University of North Carolina-Chapel Hill, Department of Pathology and Laboratory Medicine CB \#7525, \\ Glaxo Research Laboratories Rm 148, Chapel Hill, NC 27599-7525, USA
}

Received 17 May 2005; accepted 26 August 2005

\begin{abstract}
Previous studies with alcohol-associated malondialdehyde-acetaldehyde (MAA)-modified proteins have demonstrated an increase in the expression of adhesion molecules, and the secretion of pro-inflammatory cytokines/chemokines by rat sinusoidal liver endothelial cells (SECs). However, no studies have been initiated to examine the effects of MAA-modified proteins on the expression of the extracellular matrix (ECM) protein, fibronectin and its isoforms. For these studies, SECs were isolated from the liver of normal rats, and exposed to MAA-modified bovine serum albumin (MAA-Alb). At selected time points, the total plasma and cellular fibronectin were determined by Western blot. Injection of rat liver via the mesenteric vein with MAA-Alb was performed in an effort to evaluate the potential in vivo role of MAA-modified proteins in the development of fibrosis. Expression of both plasma and cellular fibronectin was significantly increased over controls in the MAA-Alb stimulated SECs (>3-fold). Importantly, the isotype of fibronectin secreted was determined to be of the EIIIA variant and not EIIIB. These data were confirmed using RT-PCR procedures on liver tissue from; isolated SECs, and from an in vivo animal model wherein MAA-Alb was administered via the mesenteric vein. Thus, these studies demonstrate that MAA-modified proteins initiate a pro-fibrogenic response by initiating the expression of the fibronectin EIIIA isoform by SECs.
\end{abstract}

Keywords: Adducts; Aldehydes; Fibronectin; Extracellular matrix (ECM); Alcohol; Endothelial cells

\section{Introduction}

The sinusoidal endothelial cells (SECs) that line the blood vessels in the liver play a key role in the process of

Abbreviations: Alb, bovine serum albumin; IL-1 $\beta$, interlukin-one beta; $\mathrm{KCs}$, Kupffer cells; LPS, lipopolysaccharide; MAA, malondialdehydeacetaldehyde-adduct; SEC, sinusoidal liver endothelial cells; TGF- $\beta$, transforming growth factor-beta

All work was performed at the Veterans Administration Alcohol Research Center, Omaha Veterans Administration Medical Center, Research Service 151, Rm 321, 4101 Woolworth Avenue, Omaha, NE 68105, USA.

* Corresponding author. Present address: Omaha VA Medical Center, Research Service 151, Rm 321, 4101 Woolworth Avenue, Omaha, NE 68105, USA. Tel.: +1 402346 8800x3550; fax: +1 4024490604 .

E-mail address: GTHIELE@unmc.edu (G.M. Thiele). hepatic fibrogenesis that follows liver injury. The ability of SECs to react to a stimulus by the release of fibronectin and subsequently activate stellate cells makes them essential to the wound healing process [1,2]. Alcohol is a known hepatotoxin that has been shown to cause severe damage to the liver and increase liver fibrogenesis [3-6]. Metabolic byproducts of alcohol metabolism (acetaldehyde and malondialdehyde) can bind to proteins and form stable adducts, which have been known to cause many deleterious effects on various cells of the liver [7-10]. The ability of malondialdehyde and acetaldehyde to react with proteins to form the stable hybrid adduct (MAA) [11] has profound implications in the onset of alcoholic liver disease [12]. The MAA-adduct has been shown to cause the release of cytokines/chemokines by SECs, KCs, and stellate cells 
[13-15]. It is known that cytokines, growth factors, hormones, and enzymes are present in their inactive form and upon activation, can alter the ECM and initiate the fibrogenic process [16]. The MAA-adduct has been found in the serum of alcohol-induced cirrhosis and hepatitis patients and rats chronically consuming alcohol $[12,17]$.

The extracellular matrix (ECM) of the liver is comprised of many complex molecules that work together to maintain structural coherence between the cells. While this is one of the major functions of the ECM, it also modulates other biological functions, which include cell proliferation, migration, differentiation, and gene expression in concert with numerous growth factors, hormones, enzymes, and cytokines [18]. Components of the ECM, including collagen, glycoproteins, and proteoglycans, may be increased 3-5fold upon injury to the liver [19]. Once the liver is injured, pro-inflammatory, and pro-fibrogenic cytokines are released by Kupffer $(\mathrm{KC})$, and sinusoidal endothelial cells, resulting in the recruitment and activation of immune cells, an increase in ECM proteins, and the subsequent repair of the liver.

Fibronectin release by SECs is involved in the initiation of the process of fibrogenesis in that it is one of the earliest ECM components expressed in the liver following injury $[2,20]$. This was demonstrated using bile duct ligation as a model of liver injury, where all the individual cells of the liver were isolated and tested for expression of EIIIA and EIIIB splice variants. It was found that the greatest quantity of fibronectin was found in the SECs, which consisted mostly of the EIIIA variant. The EIIIB variant, after liver injury, was found to be localized in the lipocytes and not the SECs. While an important product in the wound healing process, EIIIB shows up later on in the repair process. Deposition of the EIIIA variant by injured SECs was capable of activating stellate cells in an in vitro assay, which caused the release of type IV collagen [2]. Therefore, it was the purpose of this study to determine if MAAadducted proteins stimulate SECs to increase the synthesis of the EIIIA fibronectin splice variant.

\section{Materials and methods}

\subsection{Rats}

Male Wistar rats were obtained from the National Cancer Institute and maintained on Purina rat chow diet until sacrificed. All procedures were approved by the animal subcommittee of the Omaha VA Medical Center (accredited by American Association for the Accreditation of Laboratory Animal, AAALAC) and were in accordance with guidelines of the National Institutes of Health (1985).

\subsection{Chemicals and proteins}

Bovine serum albumin (Alb) and fibronectin were purchased from CalBiochem (La Jolla, CA). Acetaldehyde
(AA) was obtained from Aldrich Chemical Co. (Milwaukee, WI). Malondialdehyde (MDA) was obtained as the sodium salt $(\mathrm{MDA} \sim \mathrm{Na}$ ) by treatment of tetramethoxypropane (Aldrich Chemical Co.) with $\mathrm{NaOH}$, according to the method of Kikugawa and Ido [21]. Lipopolysaccharide (LPS), phytic acid (PA), and diethylenetriaminepentaacetic acid (DTPA), transforming growth factor-beta (TGF- $\beta$ ), interlukin-1 beta (IL-1 $\beta$ ), and Percoll were purchased from Sigma Chemical Co. (St. Louis, MO). Rat liver cytosol was isolated by ultracentrifugation methods previously reported [22].

\subsection{Preparation and labeling of ligands}

Bovine serum albumin-MAA (MAA-Alb) or liver cytosol-MAA (MAA-cytosol) was prepared by reacting $1.0 \mathrm{mM}$ AA and $2.0 \mathrm{mM}$ MDA with $30 \mu \mathrm{M}$ bovine serum albumin or rat liver cytosol in $0.1 \mathrm{M}$ phosphate buffer $\mathrm{pH}$ 7.2, containing $2 \mathrm{mM}$ DTPA and $2 \mathrm{mM}$ PA at $37^{\circ} \mathrm{C}$ for 3 days, followed by dialysis against three changes of $0.1 \mathrm{M}$ sodium phosphate buffer for $24 \mathrm{~h}$ at $4{ }^{\circ} \mathrm{C}$ [11]. Modified protein concentrations were determined using the BCA protein assay from (Pierce, Rockford, IL) and diluted to 10 or $25 \mu \mathrm{g} / \mathrm{ml}$ depending on the assay.

\subsection{Isolation of liver sinusoidal endothelial cells}

Liver sinusoidal endothelial cells were prepared by perfusion and differential centrifugation methods as described previously $[23,24]$. Briefly, following isolation cells were counted on a hemocytometer and the viability was determined by trypan blue exclusion, and found to be greater than $85 \%$ viable. SEC purity was assessed by staining with antibodies specific for the various cell types of the liver using a Fluorescent Activated Cell Scanner (FACSCalibur, San Jose, CA) as previously reported [13]. SECs were determined to be $>90 \%$ pure.

\subsection{MAA-Alb stimulation of SECs}

SECs from chow-fed rats were plated on 24 well plates coated with collagen IV and incubated at $37{ }^{\circ} \mathrm{C}$. After $24 \mathrm{~h}$ of incubation, the cells reached confluency, were washed with 10\% M199/F12 and stimulated with media only, $25 \mu \mathrm{g} / \mathrm{ml} \mathrm{Alb,} 25 \mu \mathrm{g} / \mathrm{ml}$ MAA-Alb, $5 \mathrm{ng} / \mathrm{ml} \mathrm{IL-1}$ $\beta, 10 \mu \mathrm{g} / \mathrm{ml}$ LPS, and $2 \mathrm{ng} / \mathrm{ml}$ TGF- $\beta$. SECs were incubated overnight, supernatants collected and frozen at $-70{ }^{\circ} \mathrm{C}$. The cells were washed with Dulbecco's phosphate buffed saline (D-PBS) and lysed with $2 \%$ reduced (0.5 M 2-ME) sample buffer for use in Western blot experiments.

\subsection{Determination of soluble total fibronectin}

Supernatants were assayed for soluble fibronectin using an inhibition ELISA. Briefly, 96-well ELISA plates 
(Immulon 4 HBX, Thermo Labsystems, Franklin, MA) were coated with $200 \mathrm{ng} /$ well of rat plasma fibronectin (Calbiochem, San Diego, CA) in bicarbonate buffer $(\mathrm{pH}$ 9.6) and incubated at $4{ }^{\circ} \mathrm{C}$ overnight. In $0.5 \mathrm{ml}$ RIA vials, supernatants and rat plasma fibronectin standard were mixed with rabbit anti-rat fibronectin (Calbiochem) in phosphate-buffered saline containing $0.05 \%$ Tween 20 (PBST) at a dilution of 1:5000 and incubated overnight at $4{ }^{\circ} \mathrm{C}$. The plate coated with fibronectin was washed with PBST and blocked with $2 \%$ Alb for 30 min. Supernatant and the fibronectin standard were transferred to the fibronectin-coated plate and incubated at room temperature for $2 \mathrm{~h}$. Plates were washed with PBST, alkaline phosphatase goat anti-rabbit IgG (H\&L) (Zymed Laboratories, San Francisco, CA) was added, and the plate was incubated for $1 \mathrm{~h}$ at room temperature. After washing with PBST, $p$ nitrophenyl phosphate substrate (Sigma) was added and absorbency detected by a Dynatech MicroELISA Reader MR7000 (Dynatech, Chantilly, VA) at $410 \mathrm{~nm}$. Data were determined by extrapolation from a fibronectin standard curve and calculated using Biolinx Software (Dynatech).

\subsection{Determination of cellular fibronectin and splice variants}

Cell lysate total protein was determined by the BCA protein assay (Pierce). The protein concentration in each sample was adjusted to $50 \mu \mathrm{g} / \mathrm{ml}$ and electrophoresed in precast 4-15\% SDS polyacrylamide gradient gels (BioRad, Hercules, CA) in the presence of sodium dodecyl sulfate (SDS-PAGE). The proteins were then transferred onto immobilon PVDF membranes (Millipore, Billerica, MA) overnight using a wet mini-transblotter (BioRad). To determine the presence of total cellular fibronectin, blots were blocked with $2 \%$ milk in PBST and incubated with rabbit anti-rat fibronectin (Calbiochem) at a dilution of 1:1000 overnight at $4{ }^{\circ} \mathrm{C}$. A $1-50,000$ dilution of HRP conjugated goat anti-rabbit IgG (Sigma) was used to detect the primary antibody and the HRP detected by ECL (Amersham, England). Gel bands were quantified using SigmaGel (Jandel Scientific, 2002) and protein size determined using molecular weight standards from (BioRad) and a positive total fibronectin control.

In order to determine EIIIA and EIIIB splice variants, cell lysates were electrophoresed and blotted as described above. Splice variants were assayed for by using the antibodies to mouse anti-cellular fibronectin (cFn) monoclonal (Clone DH1) EIIIA specific (ICN Biomedicals, Inc., Aurora, $\mathrm{OH})$ at a dilution of 1:1000 and mouse antihuman fibronectin III-15 monoclonal antibody EIIIB specific (Chemicon International, Inc., Temecula, CA) at a dilution of 1:1000. Detection of the primary antibody was determined by HRP goat anti-mouse $\operatorname{IgG} 1: 50,000$ (Sigma) and autoradiography using ECL (Amersham, England).

\subsection{Rat mesenteric vein injection of modified proteins}

Chow-fed rats were subjected to surgery in order to isolate the mesenteric vein for injection. Prior to surgery, rats were fasted for $2 \mathrm{~h}$, and then anesthetized with isoflurane. Using aseptic technique, the abdominal cavity was opened and the stomach and intestines placed on sterile gauze. The mesenteric vein was located and injected with $0.5 \mathrm{ml}$ of PBS, Alb $(500 \mu \mathrm{g}), \mathrm{MAA}-\mathrm{Alb}(500 \mu \mathrm{g})$, or sham (gut was subjected to similar handling). The mesenteric vein was allowed to clot, the bowel replaced into the abdominal cavity, and the peritoneal cavity closed with sutures and staples. Rats were watched until awake and allowed ad libitum access to food and water overnight. After $16 \mathrm{~h}$ the animals were sacrificed, the liver flushed with saline, removed, and SECs isolated as outlined above. As a positive control, a $70 \%$ partial hepatectomy was performed as previously described for liver regeneration $[25,26]$, providing a proven method of fibronectin synthesis. The excised normal tissue served as the negative control for all reverse transcriptase polymerase chain reaction (RT-PCR) experiments.

\subsection{RNA extraction and reverse transcriptase polymerase chain reaction}

RNA was extracted from whole liver and isolated SECs using an RNeasy Mini Kit per the Qiagen protocol (Qiagen, Valencia, CA). RNA was quantified and integrity verified by electrophoresis of $1 \mu \mathrm{g}$ RNA on a $1 \%$ agarose gel stained with ethidium bromide. Gel bands were detected by exposure on a Flour-S multiimager and analyzed by Quantity One software (Bio-Rad). RT-PCR procedures were performed using a Qiagen one-step RT-PCR reaction kit. Briefly, $1 \mu \mathrm{g}$ of RNA was mixed with dNTP and enzyme supplied by Qiagen with each set of primers to a final concentration of $1 \mu \mathrm{M}$. Primers used in this study (EIIIA, EIIIB, and GAPDH) were designed by Caputi et al. [25] and synthesized by Integrated DNA Technologies, Inc. (IDT, Coralville, IA). Amplifications were performed using an Eppendorf Mastercycler Personal (Brinkman, Westbury, NY) using the following cycles; denaturation $\left(1 \mathrm{~min}\right.$, at $\left.94{ }^{\circ} \mathrm{C}\right)$, annealing $\left(1 \mathrm{~min}\right.$, at $\left.56^{\circ} \mathrm{C}\right)$, extension $\left(1 \mathrm{~min}\right.$, at $\left.72^{\circ} \mathrm{C}\right)$, which were carried out for 25 amplification cycles. PCR products were analyzed by electrophoresis in $1 \%$ agarose containing ethidium bromide. Fragment size was verified using a $100 \mathrm{bp}$ DNA ladder standard (New England BioLabs, Beverly, MA) and gels exposed as described above.

\subsection{Statistical analysis}

Results are expressed as means \pm S.E.M. Statistical significance was achieved if $P$-values were less than 0.05. All statistical analysis was performed using ANOVA in SigmaStat (Jandel Scientific, 2002). 


\section{Results}

\subsection{Soluble fibronectin release in response to $M A A-A l b$}

In order to determine the ability of MAA-Alb to stimulate isolated SECs to release soluble fibronectin, SECs were isolated from chow-fed rats and incubated overnight. These isolated SECs were stimulated with either MAAmodified albumin or the appropriate positive and negative controls described in Section 2. SECs stimulated with media or Alb were tested by ELISA and shown to have an average of $300-500 \mu \mathrm{g} / \mathrm{ml}$ of soluble fibronectin (Fig. 1), while MAA-Alb caused a 2-3-fold increase to $1100 \mu \mathrm{g} / \mathrm{ml}(P<0.001)$. Similar results of MAA-Alb stimulation were observed with the positive controls IL$1 \beta(1200 \mu \mathrm{g} / \mathrm{ml})$ and TGF- $\beta(1100 \mu \mathrm{g} / \mathrm{ml})$. LPS $(500 \mu \mathrm{g} /$ $\mathrm{ml})$ stimulation of SECs had only background levels similar to that of media only or Alb.

\subsection{MAA-Alb-induced cellular and splice variant fibronectin expression}

To investigate the expression of cellular fibronectin after MAA-Alb incubation, SECs from the above experiments were washed, solublized in reducing sample buffer, electrophoresed by SDS-PAGE, blotted to immobilon paper, and stained for the presence of total fibronectin and the splice variants EIIIA/EIIIB as described in Section 2. Fig. 2 shows strong reactivity to total cellular fibronectin in SECs stimulated with MAA-cytosol $(6146 \pm 410)$ and MAAAlb $(10,325 \pm 481)$, whereas minimal reactivity was observed from the SECs stimulated with media only,

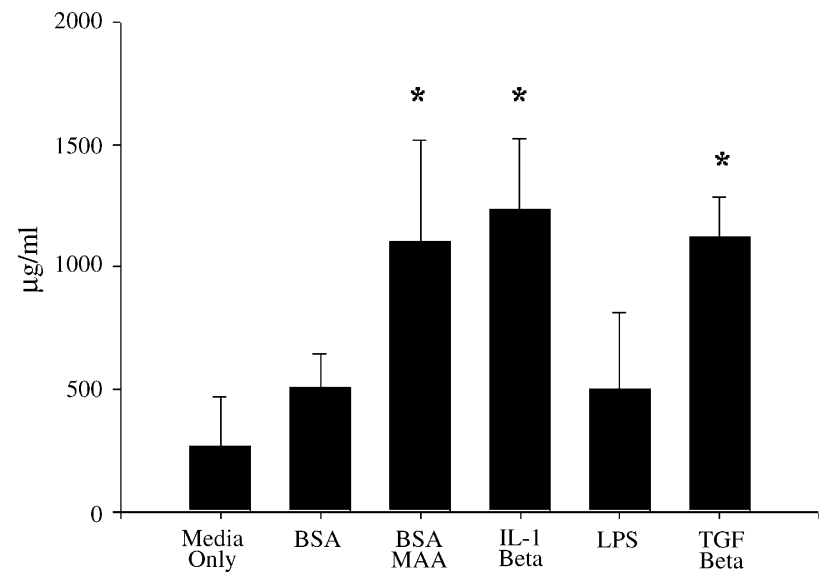

Fig. 1. Soluble fibronectin release from isolated SECs stimulated with MAA-Alb. SECs were isolated from chow-fed rats and stimulated with media only, Alb, IL- $1 \beta$, LPS, and TGF- $\beta$ overnight at $37^{\circ} \mathrm{C}$. Supernatant was collected and assayed for soluble fibronectin using an inhibition ELISA. Results indicate a 2-fold increase in fibronectin released after stimulation with MAA-Alb, comparable to that of the positive controls IL-1 $\beta$ and TGF- $\beta$. Background responses were observed when media only, $\mathrm{Alb}$, and LPS were used as the stimulating ligands. Each bar is the mean \pm S.D. of six experiments. Values significantly different from controls (Alb or media only) are indicated at ${ }^{*} P<0.001$

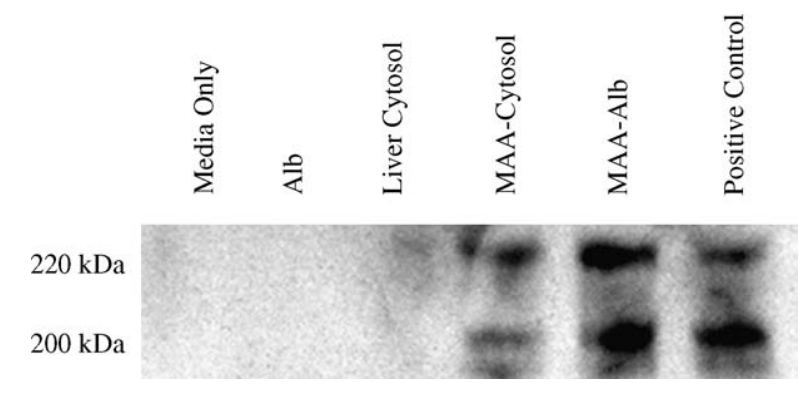

Fig. 2. Cellular fibronectin release from isolated SECs stimulated with MAA-Alb. SECs were isolated from chow-fed rats and stimulated with media only, Alb, liver cytosol, MAA-cytosol, and MAA-Alb. Western blot analysis for cellular fibronectin shows strong reactivity to the MAA-cytosol $(6146 \pm 410)$ and MAA-Alb $(10325 \pm 481)$ stimulated SECs. This was similar to the positive fibronectin control expression $(8654 \pm 855)$. No response was observed when media only, Alb, and liver cytosol were used as the ligand. Data representative of fivemals under the same conditions and were quantified by densitometry.

Alb, and unmodified liver cytosol (202 \pm 102$)$. Reactivity to the positive fibronectin control showed a density of $(8654 \pm 855)$. The fibronectin bands were present as a doublet at 200 and $220 \mathrm{kDa}$ as has been previously reported $[27,28]$.

In order to determine the presence of splice variants of fibronectin, SEC lysates from the above samples were subjected to Western blot using antibodies specific for EIIIA and EIIIB as described in Section 2. As shown in Fig. 3, when SECs are stimulated with media only or Alb (10 or $25 \mu \mathrm{g} / \mathrm{ml}$ ) there was little expression of the EIIIA variant doublet $(361 \pm 42)$ or $(1452 \pm 179)$ and no expression of EIIIB. However, when SECs were stimulated with MAAAlb (10 or $25 \mu \mathrm{g} / \mathrm{ml})$ or TGF- $\beta$ a strong increase of the EIIIA variant was observed $(5515 \pm 358)$ or $(14,331 \pm 533)$

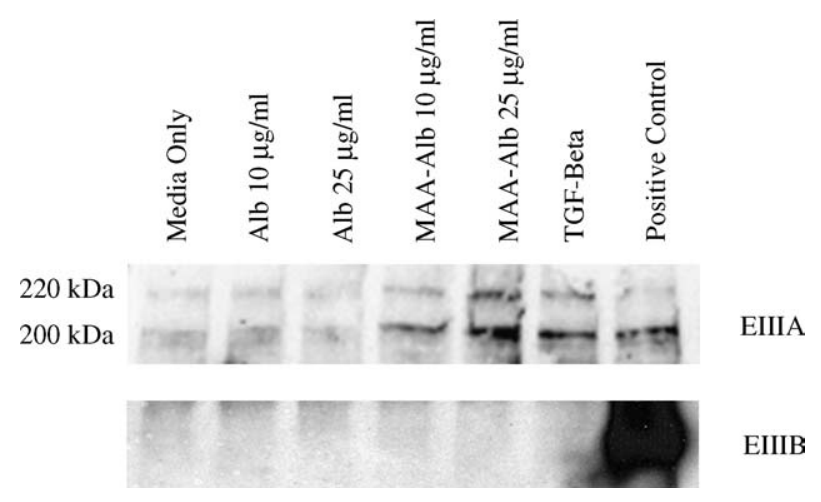

Fig. 3. Fibronectin splice variant expression from isolated SECs stimulated with MAA-Alb. SECs were isolated from chow-fed rats and stimulated with media only, Alb at 10 and $25 \mu \mathrm{g} / \mathrm{ml}$, MAA-Alb at 10 and $25 \mu \mathrm{g} / \mathrm{ml}$, and TGF- $\beta$. Western blot analysis for the EIIIA variant demonstrated a strong increase in expression when SECs were stimulated with the $10 \mu \mathrm{g} / \mathrm{ml}$ MAA-Alb $(5515 \pm 358)$ and TGF- $\beta(9708 \pm 723)$. When MAA-Alb stimulation was increased to $25 \mu \mathrm{g} / \mathrm{ml}$ EIIIA expression increased $(14,331 \pm 533)$. Small background reactivity was noted when media only or Alb $(1452+179)$ were used as the ligand. No reactivity was shown for the EIIIB splice variant, except in the positive fibronectin control. Data representative of five animals under the same conditions and were quantified by densitometry. 
and $(9708 \pm 723)$ when TGF- $\beta$ was used as a positive control. As a control on the gel, total fibronectin showed similar banding patterns and reactivity was increased $(8516 \pm 885)$. No signal for the EIIIB variant was seen after stimulation of SECs with any of the various ligands.

\subsection{RT-PCR analysis for splice variants}

In order to further demonstrate the expression of the EIIIA splice variant, RNA was isolated from SECs stimulated with $25 \mu \mathrm{g} / \mathrm{ml}$ Alb or MAA-Alb, and media for $16 \mathrm{~h}$. An increase in the expression of the EIIIA variant is represented by a band at $470 \mathrm{bp}$, while the presence of a band at $200 \mathrm{bp}$ represents the expression of the normal/ cellular fibronectin. Similarly, the EIIIB variant expresses two bands (543 and $270 \mathrm{bp}$ ). These data showed an amplification of both the EIIIA, at (200 bp), and EIIIB, at (270 bp), variant for all the stimuli (Fig. 4). However, MAA-Alb stimulation of SECs was the only ligand to initiate the increased expression of the EIIIA+ (470 bp) variant. No EIIIB+ (543 bp) variant expression was detected, and the GAPDH control was positive for the presence of quality RNA. The expression of the EIIIA and EIIIB variants is consistent with what others have observed after a $16-24 \mathrm{~h}$ incubation following partial hepatectomy [25]. Data from these sets of experiments show evidence that isolated SECs can increase fibronectin expression in vitro after MAA-Alb stimulation.

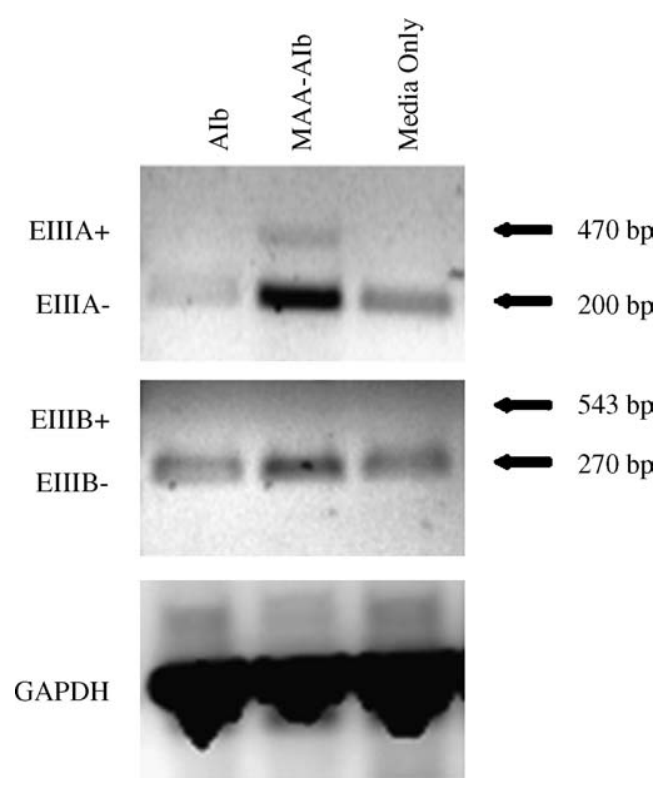

Fig. 4. RT-PCR analysis of splice variants in isolated SECs stimulated in vitro with MAA-Alb. SECs from chow-fed rats were isolated and stimulated with Alb, MAA-Alb, and media only. RNA was isolated as described in Section 2 and amplified for EIIIA and EIIIB fibronectin splice variants. Expression was observed (200 bp) for the EIIIA - variant with Alb, MAA$\mathrm{Alb}$, and media only. However, only MAA-Alb stimulation caused the expression of the EIIIA+ variant at (470 bp). EIIIB expression was detected in all samples $(270 \mathrm{bp})$. However, no expression was detected for EIIIB+ (543 bp). Gels are representative of SEC RNA from six animals stimulated with the same ligand.

\subsection{In vivo MAA-Alb mesenteric injection}

All experiments utilizing isolated SECs have an inherent problem with high backgrounds due to the non-specific activation of SECs during their isolation and culture process. This was addressed by developing an in vivo model of SEC activation. Briefly, animals were injected with the various ligands Alb (500 $\mu \mathrm{g} / \mathrm{ml})$, MAA-Alb $(500 \mu \mathrm{g} / \mathrm{ml})$, and D-PBS through the mesenteric vein as described in Section 2. After a 16-h incubation period the liver was removed and RNA isolated. As a positive control, a 70\% partial hepatectomy was performed and the regenerating tissue evaluated at the same time as the other livers [25]. RT-PCR was performed using primers specific to EIIIA, EIIIB, and GAPDH, and the products electrophoresed in $1 \%$ agarose gel. Fig. 5 shows the expression of the EIIIA (200 bp) and EIIIB (270 bp) variants for all the injected ligands. There was no expression of the EIIIB+ (543 bp) product for the negative control, any of the injected ligands or the partial heptectomy. However, there was an increase in the EIIIA+ $(470 \mathrm{bp})$ variant in the liver injected with MAA-Alb and the partial hepatectomy treatments. The GAPDH positive control was present for all the experimental conditions. PBS-injected animals show that the stress of the surgery and the vehicle itself were not sufficient to cause changes in the fibronectin expression of the liver. These data indicate that MAA-Alb affects the liver and causes the secretion of the EIIIA+ splice variant, which may contribute to the fibrogenic response.

Finally, in order to determine the involvement of SECs in this protein adduct-mediated process, chow-fed rats were injected via the mesenteric vein with Alb $(500 \mu \mathrm{g} /$ $\mathrm{ml})$, MAA-Alb $(500 \mu \mathrm{g} / \mathrm{ml})$, and PBS. Animals were

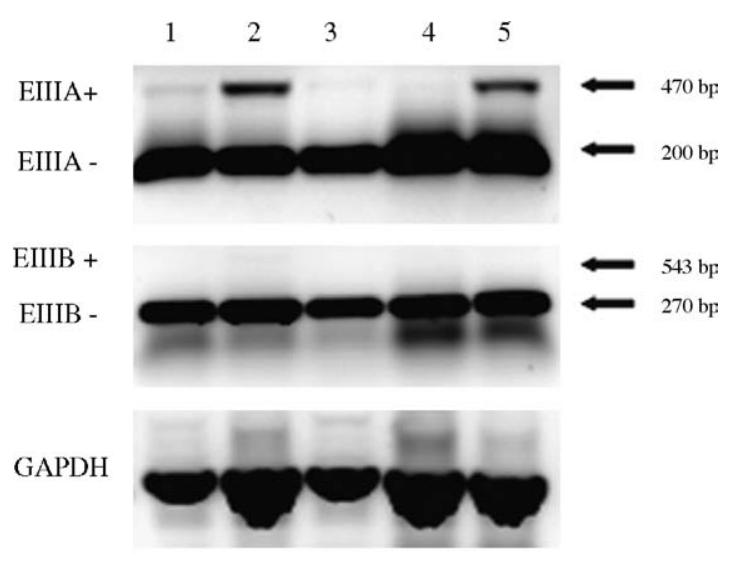

Fig. 5. Expression of EIIIA+ variant after mesenteric vein injection of MAA-Alb. Alb (1), MAA-Alb (2), or PBS (3) was injected via the mesenteric vein of chow-fed rats, incubated for $16 \mathrm{~h}$, RNA from whole liver isolated, and RT-PCR performed for EIIIA and EIIIB expression. Lane (4) represents the normal liver and lane (5) a partial hepatectomy as the positive control. Expression of the $470 \mathrm{bp}$ EIIIA+ variant was observed in the livers from animals injected with MAA-Alb, and the partial hepatectomy control. No expression was seen in the livers of animals injected with Alb, PBS, or the control liver from the partial hepatectomy. Gels are representative of five animals injected with the above ligands. 


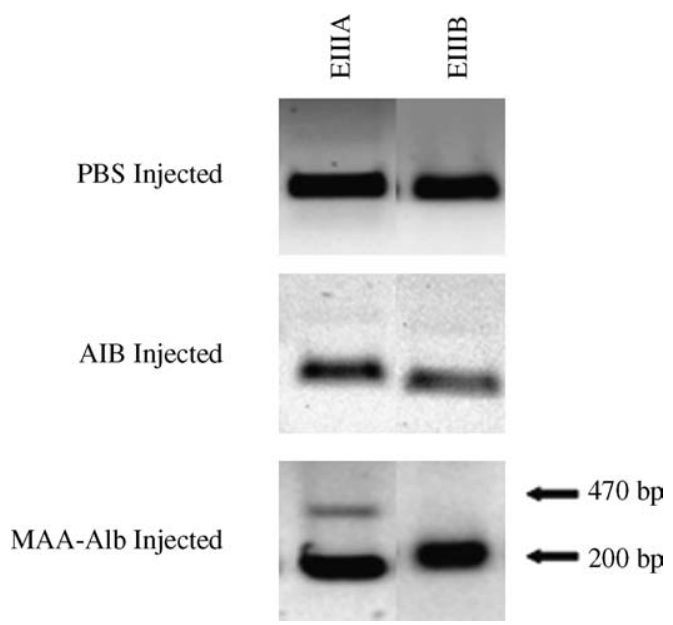

Fig. 6. Expression of EIIIA+ variant in SECs following mesenteric vein injection of MAA-Alb. Chow-fed rats were injected via the mesenteric vein with PBS, Alb, and MAA-Alb, incubated for $16 \mathrm{~h}$, SECs isolated, RNA purified, and RT-PCR performed for EIIIA and EIIIB splice variant expression. Expression of the EIIIA+ (470 bp) variant was evident in only the SECs from livers injected with MAA-Alb. Expression of EIIIB+ was negative in the SECs from livers injected with PBS, Alb, and MAA-Alb. Gels are representative of five animals injected with the above ligands.

allowed to sit overnight, the liver excised, and the SECs isolated. RT-PCR data from the isolated SECs are shown in Fig. 6. As seen in the whole liver extract data, EIIIA+ variant is only increased in the MAA-Alb injected animals and not in any of the control groups. These data demonstrate that SECs initiate the production of the EIIIA+ fibronectin variant following exposure to MAA-modified proteins in vivo. These data are consistent with those reported by Jarnagin et al. [2] using the bile duct ligation as a model of liver injury and emphasize the role of SECs in potentially mediating liver damage.

\section{Discussion}

There are numerous insults that will initiate enough damage to the liver to cause fibrosis including physical, chemical, viral, and immunological agents [29]. Ethanol is one chemical that often results in the onset of fibrosis leading to significant liver dysfunction and failure [30]. A major consequence of ethanol metabolism is lipid peroxidation, which results in the production of the aldehydic end products, 4-hydroxy-nonenal and malondiadehyde [31-33]. Another metabolic product of ethanol consumption is acetaldehyde, which is also produced in the liver, and has been shown to interact with malondialdehyde to form the stable protein adduct called malondialdehydeacetaldehyde-adduct (MAA) [11]. Antibodies to the MAAadduct have been found in the serum of patients diagnosed with alcohol-induced cirrhosis and hepatitis, and rats chronically consuming alcohol $[12,17]$. Therefore, it was the purpose of this study to determine if MAA-adducted proteins will stimulate SECs to increase the synthesis of fibronectin and/or initiate the conversion of fibronectin to the fibronectin EIIIA+ isotype.

Previous studies in our laboratory have shown the induction by MAA-adducts of pro-inflammatory cytokines/chemokines and adhesion molecules $[13,15]$. An increase in fibronectin secretion by SECs of the liver in response to MAA-adduct stimulation may be one method by which chronic alcohol consumption induces fibrosis leading to cirrhosis. In this study, the SECs from the livers of chow-fed rats exposed to MAA-Alb increased their secretion of soluble fibronectin 2-3-fold over the Alb control, which is comparable to the IL-1 $\beta$ and TGF- $\beta$ positive controls. When Western blot analysis was performed on these SECs, similar results were observed for cellular fibronectin expression. These blots demonstrated an increased expression in SECs exposed to MAA-Alb and MAA-cytosol.

It has been shown that de novo synthesis of EIIIA, a splice variant of cellular fibronectin, by stimulated endothelial cells can activate hepatic stellate cells [2]. To show that MAA-adducts may increase the expression of the EIIIA variant, SECs were exposed to MAA-Alb, and Western blots were performed using antibodies specific for EIIIA and EIIIB. Expression of the EIIIA variant was found to be increased over background in the MAA-Alb stimulated SECs and was increased with a higher concentration of the ligand. Expression of EIIIA was slightly higher in the MAA-Alb stimulated SECs when compared to the positive control stimulus TGF- $\beta$. The expression of EIIIB was not observed for all the ligands used in these studies. These Western blot studies were confirmed by RTPCR that was performed in order to assess the expression of EIIIA. Using this method it was possible to show that only the MAA-Alb exposed SECs increased the expression of the EIIIA+ variant at $470 \mathrm{bp}$. No expression of the EIIIB variant was observed under these conditions.

All studies utilizing isolated SECs in in vitro assays have inherent weaknesses. Firstly, the physical process of separation and culture in itself activates SECs, raising baseline levels of cytokine secretion that are not observed in vivo. Secondly, isolated SECs interact with soluble ligands under the non-physiological conditions of isolated cells. Removal from the extracellular matrix, cell-to-cell contact, and immediate exposure to cytokines from adjacent cells may well alter both the qualitative and quantitative responses to ligand stimulation. Because of these potential limitations, and in order to further demonstrate that MAA-adducts play a role in these processes an in vivo model was developed, wherein MAA-adducts were injected through the mesenteric vein which directly drains to the liver. These studies confirmed the findings utilizing stimulated isolated SECs and again demonstrated that MAA-modified albumin induced a substantial increase in EIIIA+ expression. The expression of the EIIIB+ variant was negative for all the experimental conditions. 
The role of SECs in the whole liver in vivo model was confirmed by isolating the SECs from the liver after ligand injection through the mesenteric vein, and demonstrating by RT-PCR that the EIIIA+ isoform was upregulated. These data demonstrated that animals injected with MAA-Alb and not the Alb or PBS controls were responsible for the increase in EIIIA+ expression. While these studies do not rule out the involvement of other hepatic cell types, this data strongly suggest that the SECs play a major role in this response.

The pathogenesis of fibrosis leading to cirrhosis involves a complex series of events [1]. Recently, it has been shown that hepatic fibrosis is a wound-healing response that occurs following chronic liver injury and is similar to what occurs in the lung, kidney, heart or skin. This observation has allowed investigators across many disciplines to share their data and advance this field significantly. In the liver, myofibroblast-like cells come from activated stellate cells. Initially, it appears that the subendothelial deposition of cellular fibronectin in the Space of Disse by sinusoidal endothelial cells provokes stellate cell activation [2,30]. The activation of resident stellate cells causes them to increase in number, degrade broken down matrix proteins, and enhance fibrogenesis by making new extra-cellular matrix components [1]. Under normal conditions, the products that activate stellate cells are removed, the liver heals and homeostasis is restored.

However, under various circumstances wherein stellate cells remain activated, then the increased production of fibril-forming matrix could lead to the loss of function by hepatocytes and reduced sinusoidal cell porosity, and eventually fibrosis and/or cirrhosis. One such mechanism that could result in the continuous stimulation of stellate cells is the release of fibronectin by SECs following exposure to MAA-modified proteins. Indeed, in this study it was shown that isolated SECs when incubated with MAA-modified proteins not only secreted both soluble and cellular fibronectin, but also the EIIIA variant which is believed to be involved in the activation of stellate cells [2]. Further studies need to be performed in order to determine if these soluble products secreted by SECs in response to MAA-modified proteins can induce stellate cells to produce extra-cellular matrices, which contribute to the development of fibrosis and cirrhosis. Regardless, these studies strongly suggest an interaction between MAA-modified proteins and SECs in the development and/or progression of alcoholic liver disease.

\section{Acknowledgments}

We thank the members of the Experimental Immunology Laboratory at the Omaha VA Medical Center including, Karen C. Easterling, Carlos D. Hunter, and Bartlett C. Hamilton III for their valuable help with this project. Supported by: National Institutes of Health Grants: R01
AA10435 and R37 AA07818. Also supported by a Department of Veterans Affairs Merit Review and the Department of Veterans Affairs Alcohol Research Center at the Omaha VA Medical Center.

\section{References}

[1] Olaso E, Friedman SL. Molecular regulation of hepatic fibrogenesis. J Hepatol 1998;29(5):836-47.

[2] Jarnagin WR, Rockey DC, Koteliansky VE, Wang SS, Bissell DM. Expression of variant fibronectins in wdfound healing: cellular source and biological activity of the EIIIA segment in rat hepatic fibrogenesis. J Cell Biol 1994;127(6 Pt 2):2037-48.

[3] Alcoholic liver disease: morphological manifestations. Review by an international group. Lancet 1981;1(8222):707-11.

[4] Tsukamoto H, Gaal K, French SW. Insights into the pathogenesis of alcoholic liver necrosis and fibrosis: status report. Hepatology 1990;12(3 Pt 1):599-608.

[5] Tsukamoto H, Matsuoka M, French SW. Experimental models of hepatic fibrosis: a review. Semin Liver Dis 1990;10(1):56-65.

[6] Chedid A, Arain S, Snyder A, Mathurin P, Capron F, Naveau S. The immunology of fibrogenesis in alcoholic liver disease. Arch Pathol Lab Med 2004;128(11):1230-8.

[7] Niemela O, Parkkila S, Bradford B, Iimuro Y, Pasanen M, Thurman RG. Effect of Kupffer cell inactivation on ethanol-induced protein adducts in the liver. Free Radic Biol Med 2002;33(3):350-5.

[8] Paradis V, Scoazec JY, Kollinger M, Holstege A, Moreau A, Feldmann $\mathrm{G}$, et al. Cellular and subcellular localization of acetaldehyde-protein adducts in liver biopsies from alcoholic patients. J Histochem Cytochem 1996;44(9):1051-7.

[9] Stewart SF, Vidali M, Day CP, Albano E, Jones DE. Oxidative stress as a trigger for cellular immune responses in patients with alcoholic liver disease. Hepatology 2004;39(1):197-203.

[10] Tuma DJ, Casey CA. Dangerous byproducts of alcohol breakdownfocus on adducts. Alcohol Res Health 2003;27(4):285-90.

[11] Tuma DJ, Thiele GM, Xu D, Klassen LW, Sorrell MF. Acetaldehyde and malondialdehyde react together to generate distinct protein adducts in the liver during long-term ethanol administration. Hepatology 1996;23(4):872-80.

[12] Rolla R, Vay D, Mottaran E, Parodi M, Traverso N, Arico S, et al. Detection of circulating antibodies against malondialdehyde-acetaldehyde adducts in patients with alcohol-induced liver disease. Hepatology 2000;31(4):878-84.

[13] Duryee MJ, Klassen LW, Freeman TL, Willis MS, Tuma DJ, Thiele GM. Lipopolysaccharide is a cofactor for malondialdehyde-acetaldehyde adduct-mediated cytokine/chemokine release by rat sinusoidal liver endothelial and Kupffer cells. Alcohol Clin Exp Res 2004;28(12):1931-8.

[14] Kharbanda KK, Todero SL, Shubert KA, Sorrell MF, Tuma DJ. Malondialdehyde-acetaldehyde-protein adducts increase secretion of chemokines by rat hepatic stellate cells. Alcohol 2001;25(2): 123-8.

[15] Thiele GM, Duryee MJ, Willis MS, Sorrell MF, Freeman TL, Tuma DJ, et al. Malondialdehyde-acetaldehyde (MAA) modified proteins induce pro-inflammatory and pro-fibrotic responses by liver endothelial cells. Comp Hepatol 2004;3(Suppl. 1):S25.

[16] Somasundaram R, Schuppan D. Type I, II, III, IV, V, and VI collagens serve as extracellular ligands for the isoforms of platelet-derived growth factor (AA, BB, and AB). J Biol Chem 1996;271(43): 26884-91.

[17] Xu D, Thiele GM, Beckenhauer JL, Klassen LW, Sorrell MF, Tuma DJ. Detection of circulating antibodies to malondialdehyde-acetaldehyde adducts in ethanol-fed rats. Gastroenterology 1998;115(3):68692. 
[18] Bedossa P, Paradis V. Liver extracellular matrix in health and disease. J Pathol 2003;200(4):504-15.

[19] Gressner AM. Hepatic fibrogenesis: the puzzle of interacting cells, fibrogenic cytokines, regulatory loops, and extracellular matrix molecules. Z Gastroenterol 1992;30(Suppl. 1):5-16.

[20] George J, Wang SS, Sevcsik AM, Sanicola M, Cate RL, Koteliansky VE, et al. Transforming growth factor-beta initiates wound repair in rat liver through induction of the EIIIA-fibronectin splice isoform. Am J Pathol 2000;156(1):115-24.

[21] Kikugawa K, Kosugi H, Asakura T. Effect of malondialdehyde, a product of lipid peroxidation, on the function and stability of hemoglobin. Arch Biochem Biophys 1984;229(1):7-14.

[22] Volentine GD, Tuma DJ, Sorrell MF. Subcellular location of secretory proteins retained in the liver during the ethanol-induced inhibition of hepatic protein secretion in the rat. Gastroenterology 1986;90(1):15865.

[23] Duryee MJ, Klassen LW, Freeman TL, Willis MS, Tuma DJ, Thiele GM. Chronic ethanol consumption impairs receptor-mediated endocytosis of MAA-modified albumin by liver endothelial cells. Biochem Pharmacol 2003;66(6):1045-54.

[24] Braet F, De Zanger R, Sasaoki T, Baekeland M, Janssens P, Smedsrod $\mathrm{B}$, et al. Assessment of a method of isolation, purification, and cultivation of rat liver sinusoidal endothelial cells. Lab Invest 1994;70(6):944-52.
[25] Caputi M, Melo CA, Baralle FE. Regulation of fibronectin expression in rat regenerating liver. Nucleic Acids Res 1995;23(2):238-43.

[26] Freeman TL, Thiele GM, Tuma DJ, Machu TK, Mailliard ME. ATA2mediated amino acid uptake following partial hepatectomy is regulated by redistribution to the plasma membrane. Arch Biochem Biophys 2002;400(2):215-22.

[27] Mueller HK, Fritsche U, Haslinger A, Landgraf R. Glucose-induced fibronectin expression in endothelial cells is mediated by protein kinase C. Exp Clin Endocrinol Diab 1997;105(1):32-8.

[28] Schwarzbauer JE, Tamkun JW, Lemischka IR, Hynes RO. Three different fibronectin mRNAs arise by alternative splicing within the coding region. Cell 1983;35(2 Pt 1):421-31.

[29] Poli G. Pathogenesis of liver fibrosis: role of oxidative stress. Mol Aspects Med 2000;21(3):49-98.

[30] Dam-Larsen S, Franzmann M, Andersen IB, Christoffersen P, Jensen LB, Sorensen TI, et al. Long-term prognosis of fatty liver: risk of chronic liver disease and death. Gut 2004;53(5):750-5.

[31] Cederbaum AI. Role of lipid peroxidation and oxidative stress in alcohol toxicity. Free Radic Biol Med 1989;7(5):537-9.

[32] Paradis V, Kollinger M, Fabre M, Holstege A, Poynard T, Bedossa P. In situ detection of lipid peroxidation by-products in chronic liver diseases. Hepatology 1997;26(1):135-42.

[33] Pietrangelo A. Iron-induced oxidant stress in alcoholic liver fibrogenesis. Alcohol 2003;30(2):121-9. 